\title{
Research on Intelligent Control System of Indoor Greening Based on Wireless Sensor Network
}

\author{
Chen Su $\mathbb{D}$ and Zhen Wang $\mathbb{D}$ \\ Hubei University of Technology, Hubei Wuhan 430068, China \\ Correspondence should be addressed to Zhen Wang; 101910943@hbut.edu.cn
}

Received 19 November 2021; Revised 31 December 2021; Accepted 7 January 2022; Published 11 February 2022

Academic Editor: Gengxin Sun

Copyright (c) 2022 Chen Su and Zhen Wang. This is an open access article distributed under the Creative Commons Attribution License, which permits unrestricted use, distribution, and reproduction in any medium, provided the original work is properly cited.

\begin{abstract}
With the acceleration of urbanization, people living in cities have a fast pace of life, great work pressure, and difficult to form a regular work and rest. As an emerging industry, indoor greening began to develop rapidly, which is of great significance to increase the area of urban green space and improve the living environment in a specific range. Aiming at the contradiction between urban resources and demand, the existing indoor greening automatic management system is studied, and an indoor greening intelligent management system based on wireless sensor network is designed. After careful study of indoor greening and wireless sensor technology, the design scheme is obtained by comprehensively considering the factors of intelligent management and resource saving. The hardware and software are designed from four parts: acquisition end, control end, gateway, and host computer. The plant growth environment parameters such as light intensity are obtained in real time. Using analytic hierarchy process and fuzzy comprehensive evaluation method to evaluate and analyze the plant growth environment, it can collect the relevant data of light, temperature, humidity, gas, and soil in real time and transmit the collected data to the host computer through wireless sensor network. The quantitative environmental suitability index is put forward, and the intelligent management of indoor greening plants is realized through the feedback control system. This topic combines wireless sensor technology with indoor greening, which can provide a new application field for the existing indoor greening. Once mature products are launched into the market, it can save time and energy for people to raise plants and better beautify and optimize the living environment.
\end{abstract}

\section{Introduction}

Indoor greening refers to the use of foliage plants and other ornamental materials to break the monotony of the indoor environment, adjust the indoor temperature and humidity, purify the air, and create a vibrant, beautiful, and comfortable indoor environment. Indoor greening mainly adopts three types of plants, namely, foliage plants, bonsai, and potted flowers [1]. The leaf of foliage plants is the main ornamental part, or the leaf shape is strange, or the leaf color is beautiful and has low requirements for sunlight and simple management, which is very popular all over the world [2]. Bonsai is a traditional Chinese art with high ornamental value, but it needs careful maintenance and high cost. It is mostly used for rent. Potted flowers have good decorative effect. Because they have high requirements for light and need more care, they are mostly used in festivals to set off the festival atmosphere. Indoor greening can absorb poison gas, relieve mood, and beautify the room, and the indoor space has the function of reconstruction [3]. Indoor plants can absorb harmful toxic gases emitted by household appliances and plastic products, such as carbon monoxide and nitrogen peroxide. Indoor plants can make people feel relaxed, regulate people's optic nerve and heart rhythm, and alleviate neurological fatigue. Indoor greening can beautify the room, make the room present the beauty of artistic conception and art, and make the indoor atmosphere more flexible [4]. It is an indispensable part of indoor decoration. The size and height of indoor plants can adjust the sense of proportion of space, and the flower walls, flower pools, and potted plants placed indoors can separate the room into small spaces that are both 
transparent and slightly hidden, so as to achieve the effect of mutual integration [5]. Naturally, people living in downtown areas yearn for a comfortable, quiet, natural, and harmonious living environment, so they begin to greening the environment to varying degrees [6]. As an emerging industry, indoor greening began to develop rapidly, which is of great significance to increase the area of urban green space and improve the living environment in a specific range. In recent years, some cities have frequently held family greening and decoration design competitions, family gardening seminars, exchange meetings, and other activities, which have greatly inspired people's enthusiasm for family gardening activities [7].

Since the 21st century, wireless sensor network (WSN) has developed rapidly under the leadership of wireless communication technology. WSN network researchers have also successfully developed micro sensor nodes with low power consumption, low cost, and wireless communication capability. Therefore, it is possible to build a low-cost WSN network. At the same time, with the development and progress of society, WSN network has a significant application demand in the fields of ecological protection, energy conservation and emission reduction, and modern services [8]. People's application needs for the network show diversity and continue to move closer to the direction of personalization and intelligence. The current network structure has the coexistence of a variety of heterogeneous network coverage and complex and diverse access technologies. The limitations of this network organization structure are constantly expanding, making heterogeneous network interconnection, that is, heterogeneous network integration, become the main direction of the development of the next generation network. The interconnection and interworking of heterogeneous networks and the cooperative work of network nodes are also an important topic that must be solved in the research process of next generation networks [9]. At present, the problems of small coverage and limited network capacity are gradually emerging in wireless networks. The emergence of heterogeneous wireless network integration technology not only solves the limitations of existing networks but also becomes an effective means for network terminal nodes to provide network access services anytime and anywhere and mobile computing power. Moreover, this technology has attracted the continuous attention of people from all walks of life [10]. It has great development potential in various application fields in the future. As a new generation of wireless network technology, wireless sensor network integrates computer technology, wireless communication technology, intelligent embedded technology, and sensor technology. In the monitoring area, the staff scatter the sensor nodes freely [11]. These sensor nodes are automatically built into a WSN network through self-organization. The network can complete the collection, transmission, processing, and transmission of the physical parameters of the perceived objects in the monitoring area [12]. The WSN wireless sensor network finally transmits the perceived physical information to the host computer monitoring software and provides decision support for users. Because the sensor nodes scattered in the monitoring area can form communication links through self-organization, WSN network does not have high requirements for infrastructure construction. Even without infrastructure construction, nodes work together to complete the information acquisition process of sensing objects [13]. A large number of sensors deployed in the monitoring area are single nodes. In addition to the functions of sensing object information acquisition and data processing, it can also process the information transmitted from adjacent nodes for simple dump and processing [14]. However, due to the limitations of low microprocessor processing rate, limited storage space, and limited energy supply, the communication ability of sensor nodes is relatively weak [15]. Therefore, we cannot rely on a single sensor node to complete the task of collecting, processing, and transmitting the information of the monitoring area, so we need to cooperate with adjacent nodes to process the information of the sensing object and finally transfer the sensing object information to users to provide decision support [16]. Intelligent control is a control mode with intelligent information processing, intelligent information feedback, and intelligent control decision-making. It is an advanced stage of the development of control theory. It is mainly used to solve the control problems of complex systems that are difficult to be solved by traditional methods. The main characteristics of intelligent control research object are uncertain mathematical model, high nonlinearity, and complex task requirements.

Through research and practice, this paper applies wireless sensor technology to indoor greening and develops indoor greening application module in command and control system. In addition, this paper also uses wireless sensor technology to develop a convenient, intelligent and cost-effective maintenance management control system suitable for family greening, so as to realize the intelligent management and control of humidity, light, temperature and landscape lights.

The rest of this article is organized as follows. Section 2 discusses related work. Section 3 introduces the experimental method. Section 4 analyzes the results. Section 5 gives the conclusion.

\section{Related Work}

Indoor greening and decoration refer to the use of ornamental materials based on indoor foliage plants in accordance with the characteristics of the indoor environment, combined with people's daily needs, to beautify the utensils and places used. This kind of beautification decoration is based on the needs of people's material and spiritual life. The entire indoor environment is designed, decorated, and arranged to integrate indoor and outdoor, reflecting the combination of dynamic and static and achieving the integration of people, indoor environment, and nature. Harmony and unity, it is an important breakthrough in traditional architectural decoration. Indoor greening and decoration refer to the use of ornamental materials based on indoor foliage plants in accordance with the characteristics of the indoor environment, combined with people's daily needs, to beautify the utensils and places used. This kind of beautification decoration is based on the needs of 
people's material and spiritual life and is designed, decorated, and arranged in line with the entire indoor environment, so that indoor and outdoor are integrated, reflecting the combination of dynamic and static and reaching people, indoor environment, and nature. It is an important breakthrough in traditional architectural decoration. In the field of agriculture, wireless sensor networks can be used to monitor the environment and soil moisture in orchards, vegetable gardens, greenhouses, greenhouses, and other places in real time, so as to realize the functions of automatic collection, wireless transmission, real-time monitoring, network release, and remote monitoring of soil moisture information in farmland, orchards, and nurseries and formulate management plans for producers $[17,18]$. Wireless sensor network can quickly and effectively obtain soil moisture information and transmit information with low cost and high reliability, which is helpful to scientific planting and improve yield [19]. Air humidity, temperature, ambient light intensity, $\mathrm{CO} 2$ concentration, water content in soil, $\mathrm{pH}$ value, etc. are important parameters for monitoring [20]. At present, researchers have developed and designed a variety of wireless sensor network monitoring systems using different technologies and methods to monitor the field, orchard, vegetable greenhouse, and greenhouse environment in real time [21]. Si and Yang [22] developed the remote monitoring system of citrus orchard soil and realized the remote and real-time monitoring of citrus orchard by using XBee PRO module with ZigBee wireless data transceiver. Shao et al. [23] developed and designed a low-power greenhouse monitoring system based on ZigBee, which can accurately collect environmental information such as illumination, air temperature and humidity, soil moisture, and carbon dioxide concentration in the greenhouse and well avoid the problems of complex construction, many lines, and difficult maintenance in the traditional greenhouse wired monitoring system [24]. Alsamhi et al. [25] use wireless sensor network to collect soil moisture content in each irrigation area of farmland, analyze and process the collected data, and formulate water use plan by using correct prediction and decision-making methods. Agricultural irrigation accounts for $70 \%$ of China's total water consumption. Wireless sensor network realizes automatic water saving and energy saving through environmental monitoring and can build an efficient, low energy consumption, low investment, and multifunctional agricultural water-saving irrigation platform. Jing et al. [26] studied farmland information collection and automatic drip irrigation based on ZigBee technology and implemented zoned precision irrigation on farmland by collecting information such as plant soil humidity, ambient temperature, and light intensity. Odiyur et al. [27] used ZigBee wireless communication technology to design the garden automatic water-saving irrigation system, which can collect the soil temperature and humidity in the garden and control the sprinkler and drip irrigation valves according to the temperature and humidity. Bai et al. [28] used GSM network to send short message instructions through mobile phone to realize micro irrigation remote control of garden ornamental plants. Mobile phone keys or short message instructions can start and stop irrigation. Han et al. [29] used ZigBee wireless sensor network and GPRS network to design and develop a water-saving irrigation control system based on CC2530 and MSP430 to automatically carry out farmland watersaving irrigation according to the collected soil moisture content information.

\section{Experimental Method}

3.1. Principles of Indoor Greening and Construction of Wireless Sensor Network. The automatic maintenance system of indoor greening is different from the widely used farmland intelligent irrigation system and the artificial climate room used in the laboratory. Although they all control the three factors of light, humidity, and temperature, they also pay attention to the requirements of multiple systems due to different application fields and purposes. Farmland conservation focuses on income, and laboratory scientific research conservation focuses on accuracy. However, indoor greening is based on the premise of facilitating home and improving the living environment. The maintenance system needs to be integrated with the home environment to meet people's needs in beauty and convenience. In order to make rational use of resources, the accuracy control can be relaxed appropriately. Many horticultural lovers like plants, but they do not easily try to raise plants. Many people move plants from flower shops to their homes and soon dry up. Some people can feed for a few months, while others can only feed for a week or two. Raising plants is a difficult problem for many people. First, people lack plant knowledge.

In addition, plant conservation needs long-term careful care. Plants are different from pets. Pets can be called and spoiled. Plants are different. They only grow quietly and are easy to be ignored. The pace of modern life is too fast, people's energy is very scattered, and it is easier to forget to maintain the flowers and plants at home. Although many people clearly know the benefits of plants to mankind, they still flinch. Therefore, the first problem to be solved by the maintenance system is to realize automatic maintenance, share worries for growers, and make indoor greening simple.

The indoor greening maintenance system is applied to home life without professional maintenance, which requires the system to have high stability and long-lasting durability. Due to the wide geographical distribution of family horticulture and different climates in different regions, for example, families in tropical areas do not need to consider the problem of cold protection, and there is no need to take lighting supplement measures for maintenance under full light.

The planting sites are free to play by the head of household, and the climatic environment is different. Different families carry out horticultural activities on different scales, and their living areas are large and small, and some species are few. Only plants are planted on the balcony, with small area and concentration. Some are arranged with greening on the villa roof, courtyard, and indoor. The area to be managed is large and scattered. This requires that the system has different configurations, and the acquisition end and control end can be expanded and reduced according to the needs to adapt to different home gardening scenarios. Therefore, the 


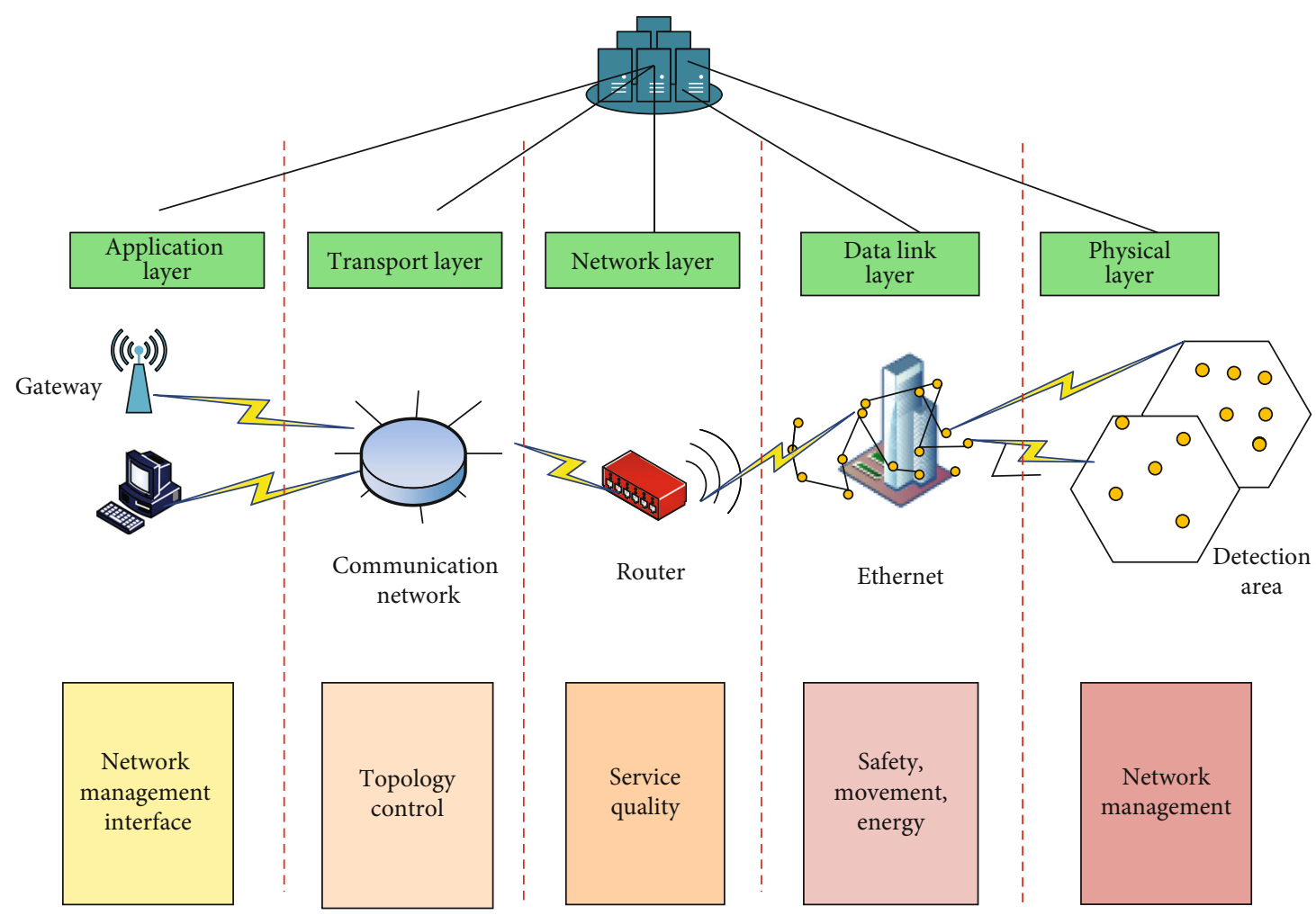

FIgURE 1: Network structure diagram of wireless sensor.

system is required to have good scalability and reassembly, so as to be applied to different home environments.

Wireless sensor network applications are characterized by low cost, low power consumption, and wide range. It is a special ad hoc network composed of sink node, task management node, and sensor node. In the monitoring area, these nodes are deployed effectively, and the physical quantities to be measured are collected, recorded, and analyzed in real time and continuously, so as to complete real-time control. Sensor node is usually a micro embedded system with relatively weak storage, communication, and data processing capabilities. Its main task is to collect data. Wireless sensor network system adopts hierarchical structure, which is composed of network management platform, network communication protocol, and application support platform. A typical wireless sensor network structure is shown in Figure 1.

\subsection{Architecture Design of Indoor Greening Intelligent} Control System. The indoor greening automatic control system is divided into five parts: acquisition terminal, control terminal, gateway, host computer, and wireless communication network. In the system, the acquisition terminal is equivalent to nerve endings, which is responsible for feeling "stimulation" and transmitting perceptual information to the host computer. Wireless communication network is equivalent to neural network, which provides a channel for the transmission of information. Gateway is the hub, which can receive and convert information and connect the host computer and other ports. The upper computer is the brain of the whole system, which is responsible for receiving signals, making final accounts and issuing commands. The control end is equivalent to muscle tissue, which is used to realize the command of the system and make practical operation. The system architecture is shown in Figure 2.

The acquisition terminal of the system includes the acquisition of three parameters: humidity, temperature, and light. The wireless sensor network adopts nRF24L01. The gateway adopts a simple gateway, which only performs data conversion without data processing. Its main function is to connect the nRF24L01 network with the host computer. The upper computer uses LabVIEW to design the virtual instrument, which is responsible for data calculation and judgment. It is a bridge between the acquisition end and the control end. The control end mainly includes watering control, cooling control, lighting control, and landscape lamp control. It also communicates with the host computer through the gateway.

3.3. Design of Acquisition Terminal of Intelligent Control System. The sensor senses physical information and generates measurable electrical signals. It is a necessary device for parameter detection in the control system. The information of various parameters in the system can be obtained through the sensor. The accuracy of the information is directly related to the measurement accuracy and control accuracy of the whole system, so as to further reasonably control the plant growth parameters according to the growth requirements of various plants, so that the plants can grow smoothly. Selecting appropriate sensors is the premise and foundation of automatic control. The information 


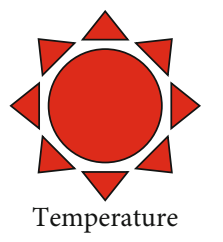

acquisition

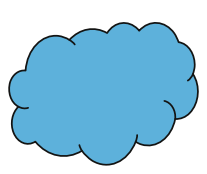

Humidity collection

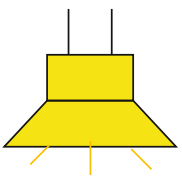

Illumination acquisition

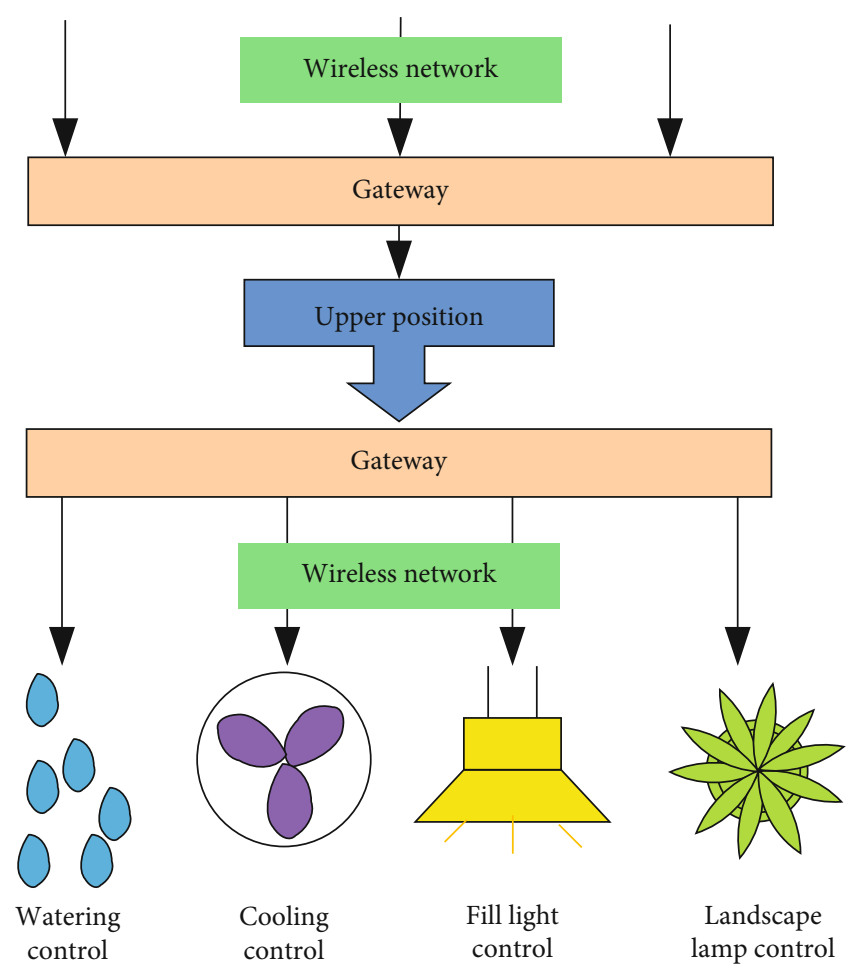

FIGURE 2: Indoor greening intelligent maintenance system structure diagram.

collected by the home gardening maintenance system mainly includes light intensity, soil humidity, and temperature. The information is collected through photosensitive sensing resistance, temperature sensor, and soil humidity sensor. The selection of the sensor at the acquisition end determines the implementation mode of the acquisition module, affects the complexity of the circuit and program, and also affects the overall performance of the system in operation.

The software design of the acquisition end is relatively simple compared with the control end. Mainly, the information can be uploaded after receiving the acquisition command of the upper computer, which can be realized through C51 programming of the single chip microcomputer. In addition, it can also realize the recognition of three types of sensing data and the processing of corresponding sensing data, including $\mathrm{AD}$ conversion and hexadecimal conversion. The outputs of soil moisture sensor and photosensitive sensor are analog signals. Before data processing, the analog signals must be transformed into digital signals for transmission and calculation. AD conversion is divided into parallel comparison type and feedback comparison type. There is no accurate digital soil moisture sensor module in the market. This design uses an ordinary

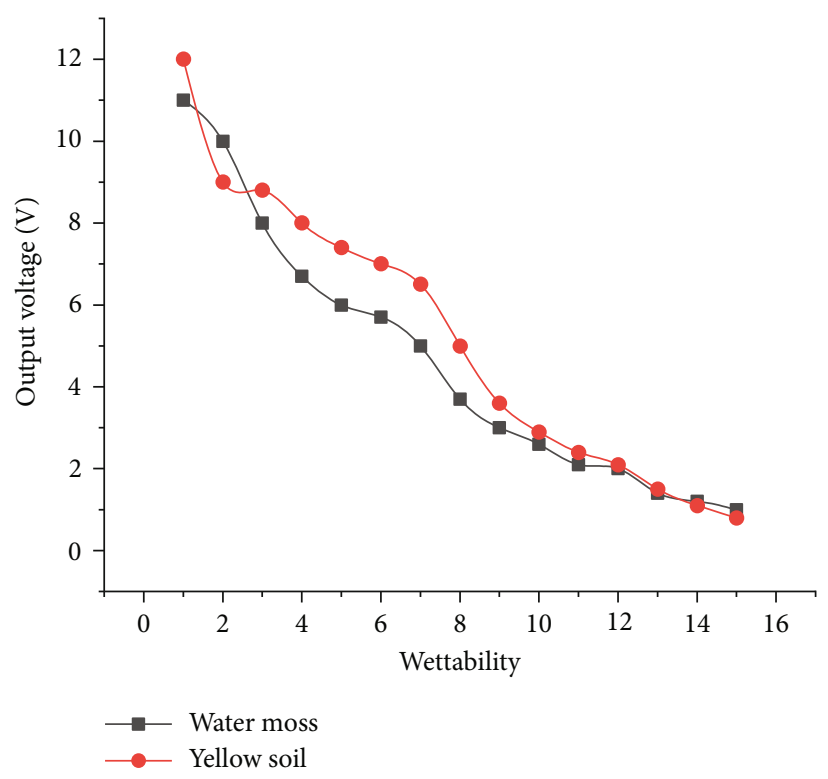

FIGURE 3: Corresponding relationship between soil humidity sensing output voltage and humidity. 


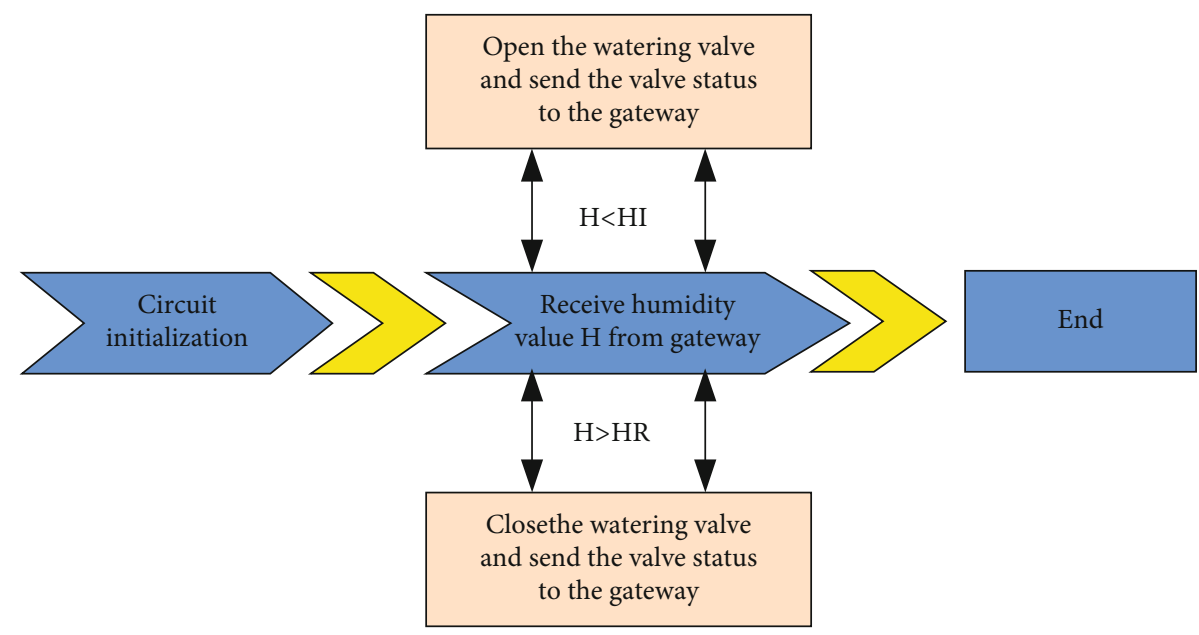

Figure 4: Flow chart of soil moisture automatic control.

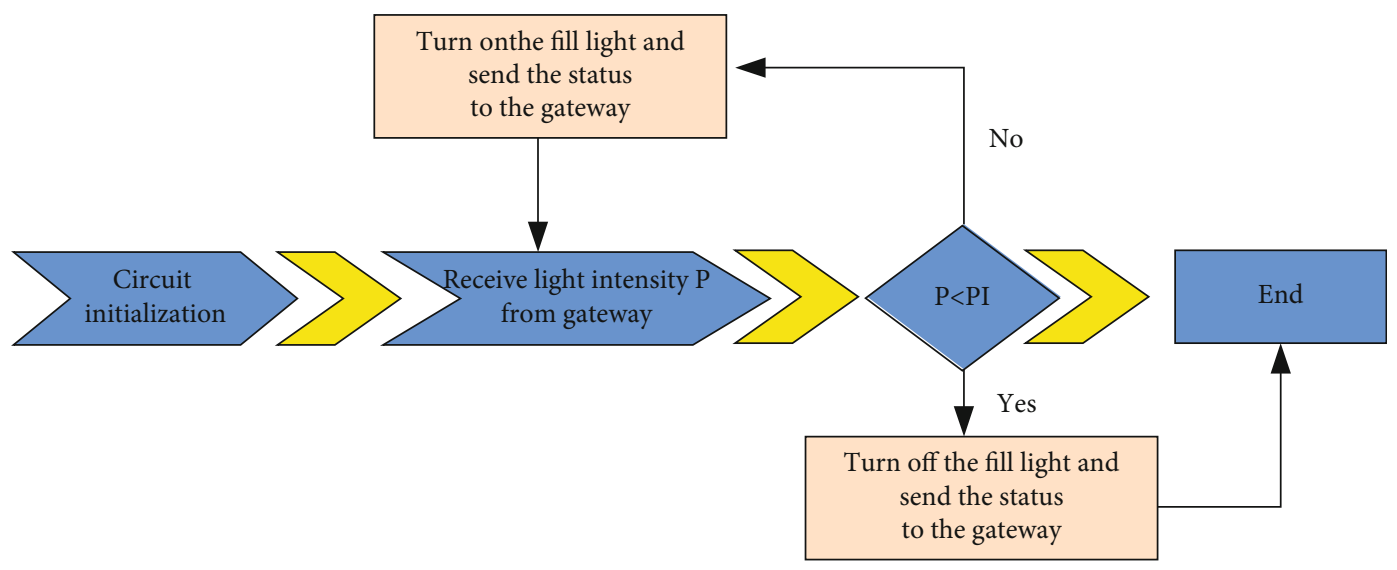

Figure 5: Flow chart of fill light control.

analog output soil moisture sensor, model yl-69. Two types of substrates, water moss and yellow soil, are used for verification. The test method is to water the dried substrate one by one, measure the sensor output every time, and observe the appearance change of the substrate until the water content of the substrate reaches saturation, and the sensor output will not change.

By detecting the moisture of two substrates of water moss and yellow soil, it is concluded that the output of yl69 soil moisture sensor is roughly the same as that of yellow soil. With the increase of humidity, the output voltage of the sensor decreases. When dry yellow soil and yellow soil are used for detection, the output voltage is $11.0 \mathrm{~V}$, which is the same as the output of detection air. When wet yellow soil and yellow soil are detected, the output voltage is less than $1.5 \mathrm{~V}$, which is the same as the output of detection pure water. As shown in Figure 3. Similarly, with the increase of the humidity of the yellow soil, the output voltage of the sensor decreases. It is observed that when the yellow soil is relatively wet, the output voltage is about $5 \mathrm{~V}$. When the output voltage drops to about $2.0 \mathrm{~V}$, the yellow soil is obviously wet, and there is water exposed at the lower part of the flower pot below $1.5 \mathrm{~V}$. Due to the different water retention between yellow soil and water moss, there are slight differences in the calibration process. For example, the water demand of yellow soil is larger, and the change of output voltage is slower.

3.4. Design of Intelligent System Control Terminal. The software design determines whether the automatic control of various functions can be realized. In the wireless transceiver protocol, watering, spraying, lighting, and landscape lighting controllers all have their own command formats. Various controllers send or receive commands in their own format to achieve control effectiveness. And each controller has a switch to set the address; that is, there can be multiple control areas. For example, if a family has a balcony and indoor green space, two watering controllers with different addresses can be used to control watering, and the upper computer can distinguish the area where the controller is located. Under specific needs, users can flexibly choose to control one or more locations. However, the maximum capacity of nodes in a system is 256 , which is sufficient for indoor greening.

Watering is the most important maintenance content of family horticulture. The biggest difference between the 


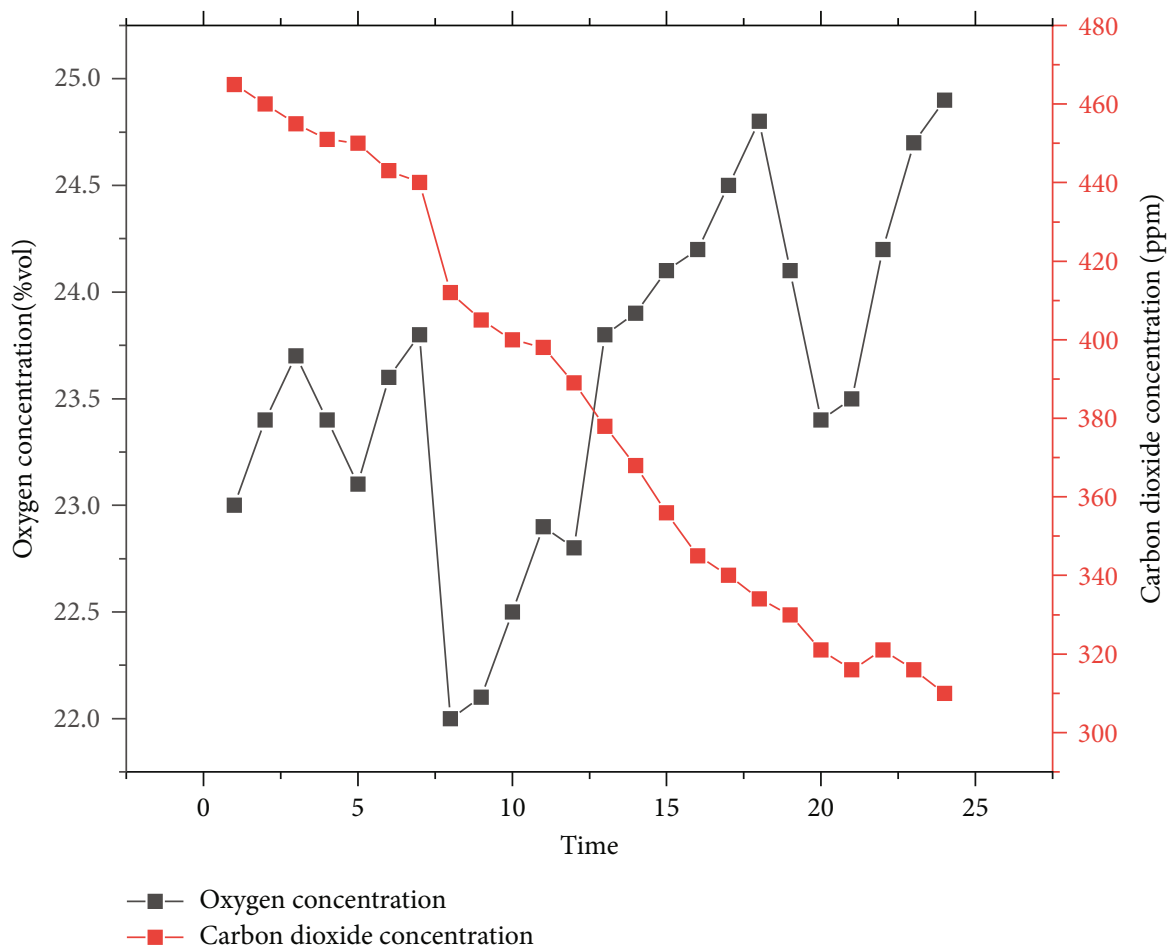

FIGURE 6: 24-hour change curve of air quality.

indoor environment and the outdoor environment is rainfall. The water in the soil of family horticulture is supplied by growers. Domestic plants are often dried or drowned under human factors. Watering is the most important maintenance content of family horticulture. The biggest difference between the indoor environment and the outdoor environment is rainfall. The water in the soil of family horticulture is supplied by growers. Domestic plants are often dried or drowned under human factors. Considering the characteristics of small home space and high requirements for environmental sanitation, the system adopts drip irrigation, which can not only save water but also prevent excess water from overflowing and soiling the room. The switch of drip irrigation is controlled by the solenoid valve, which is connected with the wireless module and the control module. The control of soil humidity is to send the humidity value collected by the sensor to the gateway through the wireless module. The gateway transmits the data to the upper computer. The upper computer compares the collected humidity value with the minimum humidity threshold. If it is lower than the minimum threshold, open the valve for irrigation until the soil humidity is higher than the maximum threshold, close the valve. Thus, the soil humidity is controlled within the more suitable range between the maximum humidity threshold and the minimum humidity threshold, as shown in Figure 4.

Light is a major limiting factor for indoor greening, and the layout and maintenance of indoor plants should be determined according to the light intensity. For example, the light in the porch and walkway is too dark, and it is difficult for general plants to survive. Greening and decora- tion can be carried out only under the condition of light supplement. The light supplement control is similar to the watering air control and cooling control in front. It also uses wireless communication technology to transmit information and commands between the acquisition end, the upper computer, and the control end, as shown in Figure 5. The upper computer compares the light intensity from the acquisition end with the minimum light intensity threshold. If it is higher than the threshold, there is no need to turn on the light; if it is lower than the threshold, it is necessary to turn on the fill light and then send the control command of the fill light to the wireless light on.

\section{Result Analysis}

4.1. Evaluation Algorithm of Indoor Greening Evaluation Factor Collection and Monitoring System. We choose an algorithm combining fuzzy comprehensive evaluation method and analytic hierarchy process. The improved comprehensive evaluation algorithm model in fuzzy comprehensive evaluation method and the index weight determined by analytic hierarchy process are used for comprehensive evaluation, which can not only avoid the loss of effective information but also realize the comprehensive measurement of operation. Through the combination of the two algorithms, we can give full play to the advantages of the two algorithms, which can not only relatively divide "good" and "bad" at the appropriate threshold and make the fuzzy phenomenon clear but also accurately measure the relative importance of the evaluation index. In this way, the combination of qualitative and quantitative can not only comprehensively consider various factors affecting 


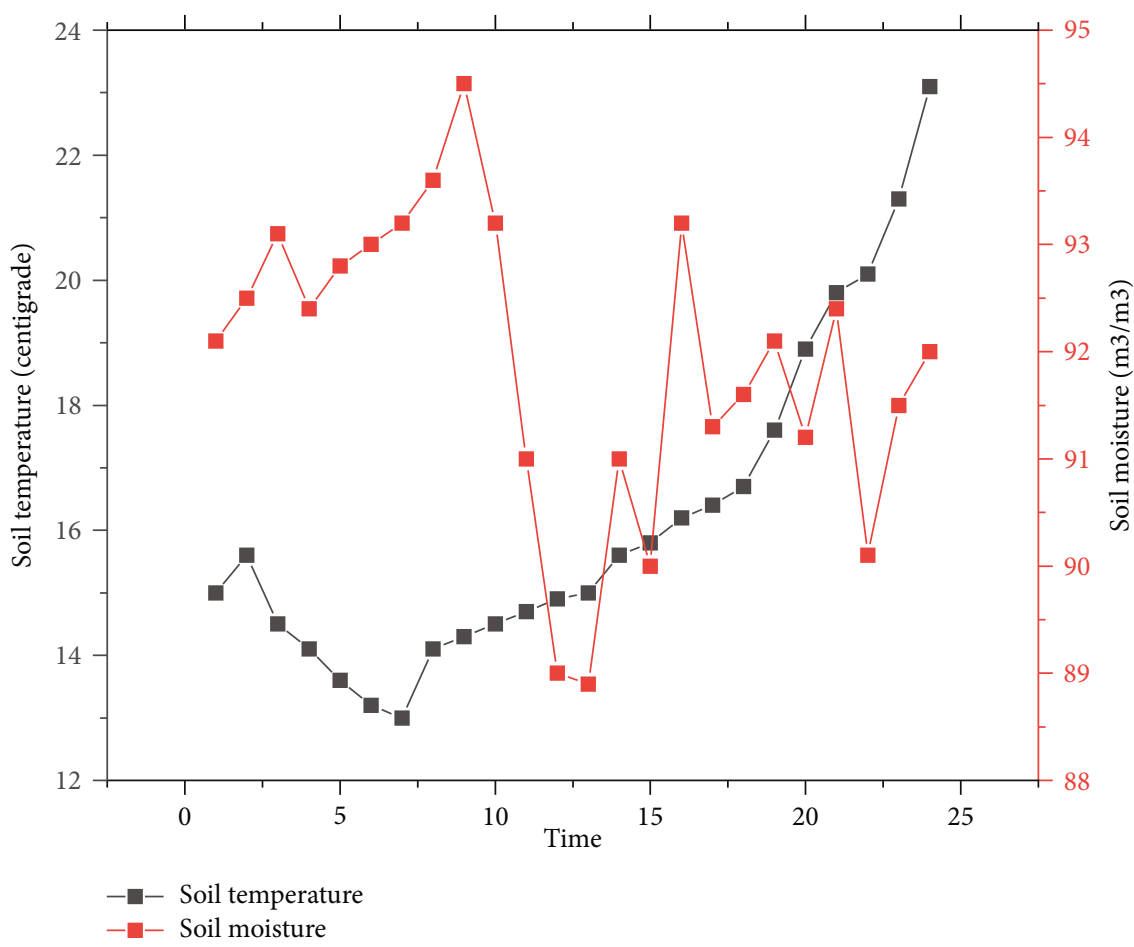

FIGURE 7: 24-hour variation curve of soil quality.

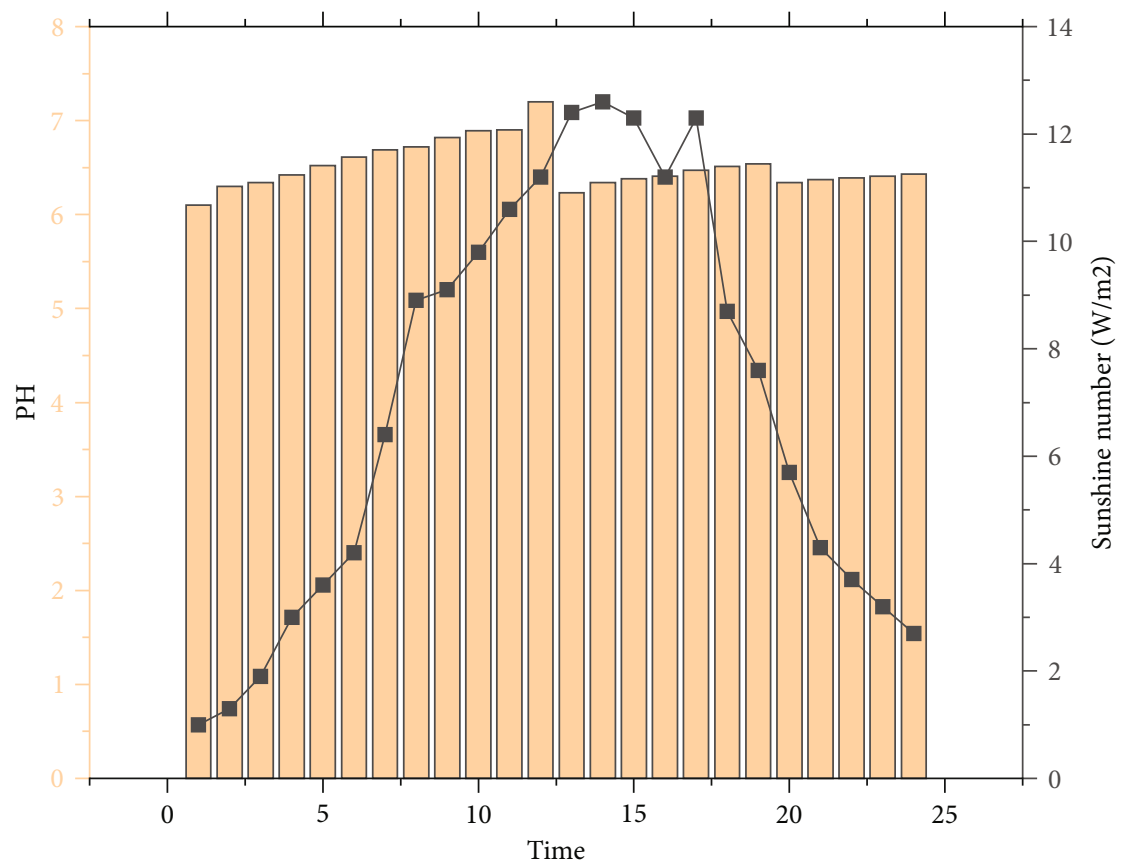

$\mathrm{PH}$

- Sunshine number

FIGURE 8: 24-hour curve of water $\mathrm{pH}$ and sunshine.

the ecological environment assessment of urban green space but also fully reflect the fuzziness of evaluation factors and evaluation process. Minimize the impact caused by personal experience or preferences, and be able to give evaluation results objectively.
4.2. Evaluation Results of Indoor Greening. The following figure shows some data obtained from the actual monitoring of the monitoring system in summer by using the indoor greening ecological evaluation factors, which provides data support for the practical application of the algorithm. 
Figure 6 shows the change curve of 24-hour air quality, in which the concentration of $\mathrm{CO} 2$ decreases continuously with the passage of time, and the concentration of oxygen fluctuates and increases. Figure 7 shows the change curve of soil quality within 24 hours. With the passage of time, the soil temperature generally increases, but from 2 o'clock to 6 o'clock, the soil temperature decreases. The soil moisture is in a relatively high state at the beginning, but with the passage of time, it is in a process of fluctuation and decline.

The improved single level comprehensive evaluation algorithm model in fuzzy comprehensive evaluation method and the index weight determined by analytic hierarchy process are used for comprehensive evaluation. It is the weight vector of four elements calculated by analytic hierarchy process, and $\mathrm{R}$ is the fuzzy set calculated by fuzzy comprehensive evaluation method. The grade of comprehensive evaluation of urban green space ecoenvironmental quality will be determined by the membership degree of each level after matrix calculation and finally depends on the grade with the largest membership value in the environmental evaluation set. Using the data collected by the system and through the calculation of the algorithm, we can conclude that the evaluation result of the selected area is excellent; that is, the air quality is good, the soil quality is good, the water quality is good, and the sunshine is moderate (Figure 8).

\section{Conclusion}

Wireless sensor networks are like the human body's sensing system, and each node is the nerve endings of the sensing system. Through the data they return, we can accurately understand the changes in the monitored area. This course applies wireless sensor network technology to indoor greening to realize intelligent remote management of indoor greening. By consulting the literature and investigating the indoor greening products and wireless sensor network technology in the market, the author has completed the design and debugging of the home gardening automatic maintenance system based on nRF24L01 wireless communication module. The main work includes hardware design and software design of acquisition end and control end, host computer program design, $\mathrm{PCB}$ proofing and welding, and system debugging. The hardware of home gardening intelligent maintenance system includes four parts: acquisition end, control end, gateway, and host computer. The acquisition end includes wireless transceiver module, three sensors (soil humidity sensor, temperature sensor, and photosensitive sensor), and data processing module. The system consists of four independent control points, which control watering, spraying, lighting, and landscape lights, respectively. Each control terminal has the same circuit structure but different programming. The hardware of control terminal includes wireless transceiver module, voltage conversion module, and data processing module. The gateway communicates with the host computer through RS232 serial port. The gateway circuit mainly includes wireless transceiver mod- ule and USB to serial port module. The system composed of wireless network has the characteristics of strong flexibility, safety, reliability, and low power consumption. It does not need manual operation, eliminates the tedious and hidden dangers of wired access, and can work stably for a long time. It is a supplement to wired control mode. This topic combines wireless sensor technology with indoor greening, which can provide a new application field for the existing indoor greening. Once mature products are launched into the market, it can save time and energy for people to raise plants and better beautify and optimize the living environment. Introducing the latest information technology into indoor greening can create convenience for indoor greening management and speed up the intellectualization of home gardening. Home gardening management technology still follows the traditional way. Management by experience and feeling requires a lot of investment in time and energy. Some irrigation timers are not common in automation. People are looking forward to a control system that can automatically manage home gardening. Although automatic management and remote control have been applied in the greenhouse with a high degree of intelligence, the system is expensive, bulky, and complex, so it is not suitable for family use. The research on the intelligent maintenance system of indoor greening based on wireless sensor network is based on the maintenance of family horticulture. It has strong applicability and is a customized maintenance system for indoor greening.

The research of indoor greening intelligent maintenance system can provide new ideas and new application fields for horticultural maintenance of smart home domain. In recent years, the Internet of Things has been widely used in home life, and smart home has emerged as an emerging industry, which can realize intelligent security system, home theater and intelligent remote control of home appliances, making life more comfortable and convenient. However, smart home does not involve gardening and maintenance. Indoor greening maintenance system can attract the interest of many smart home system developers, and it is a promising development field.

\section{Data Availability}

The data used to support the findings of this study are available from the corresponding author upon request.

\section{Conflicts of Interest}

The authors declare that they have no known competing financial interests or personal relationships that could have appeared to influence the work reported in this paper.

\section{Acknowledgments}

The study was supported by the Hubei University of Technology. 


\section{References}

[1] W. Wei, H. Song, and W. Li, "Gradient-driven parking navigation using a continuous information potential field based on wireless sensor network," Information Sciences, vol. 408, pp. 100-114, 2017.

[2] U. Gogate and J. Bakal, "Healthcare monitoring system based on wireless sensor network for cardiac patients," Biomedical \& Pharmacology Journal, vol. 11, no. 3, p. 1681, 2018.

[3] H. Li, G. Xu, and G. Xu, "Mechanical vibration monitoring system based on wireless sensor network. International Journal of Online," International Journal of Online Engineering, vol. 14, no. 6, p. 126, 2018.

[4] J. H. Seo, "Temperature data visualization for condition monitoring based on wireless sensor network," The Journal of the Korea Institute of Electronic Communication Sciences, vol. 15, no. 2, pp. 245-252, 2020.

[5] P. Song, C. Hao, and J. Wu, "Acoustic source localization using 10-microphone array based on wireless sensor network," Sensors and Actuators A: Physical, vol. 267, pp. 376-384, 2017.

[6] B. Shi, V. Sreeram, D. Zhao et al., "A wireless sensor networkbased monitoring system for freshwater fishpond aquaculture," Biosystems Engineering, vol. 172, pp. 57-66, 2018.

[7] Y. Kuo, C. L. Li, and J. H. Jhang, "Design of a wireless sensor network-based IoT platform for wide area and heterogeneous applications," IEEE Sensors Journal, vol. 18, no. 12, pp. 51875197, 2018.

[8] D. Zhang, T. Zhang, J. Zhang, Y. Dong, and X. D. Zhang, "A kind of effective data aggregating method based on compressive sensing for wireless sensor network," EURASIP Journal on Wireless Communications and Networking, vol. 2018, no. 1, 15 pages, 2018.

[9] J. Qi and G. P. Liu, "A robust high-accuracy ultrasound indoor positioning system based on a wireless sensor network," Sensors, vol. 17, no. 11, p. 2554, 2017.

[10] S. A. Kumar and P. Ilango, "The impact of wireless sensor network in the field of precision agriculture: a review," Wireless Personal Communications, vol. 98, no. 1, pp. 685-698, 2018.

[11] M. Faheem and V. C. Gungor, "Energy efficient and QoSaware routing protocol for wireless sensor network-based smart grid applications in the context of Industry 4.0," Applied Soft Computing, vol. 68, pp. 910-922, 2018.

[12] A. D. Salman, O. I. Khalaf, and G. M. Abdulsahib, "An adaptive intelligent alarm system for wireless sensor network," Indonesian Journal of Electrical Engineering and Computer Science, vol. 15, no. 1, pp. 142-147, 2019.

[13] S. Rani, S. H. Ahmed, and R. Rastogi, "Dynamic clustering approach based on wireless sensor networks genetic algorithm for IoT applications," Wireless Networks, vol. 26, no. 4, pp. 2307-2316, 2020.

[14] S. C. Chu, T. K. Dao, and J. S. Pan, "Identifying correctness data scheme for aggregating data in cluster heads of wireless sensor network based on naive Bayes classification," EURASIP Journal on Wireless Communications and Networking, vol. 2020, no. 1, 15 pages, 2020.

[15] J. Chu and W. Han, "Internet communication system protocol based on wireless sensor," Radioelectronics and Communications Systems, vol. 62, no. 8, pp. 422-429, 2019.
[16] H. Li and A. V. Savkin, "Wireless sensor networ kbased navigation of micro flying robots in the industrial Internet of Things," IEEE Transactions on Industrial Informatics, vol. 14, no. 8, pp. 3524-3533, 2018.

[17] S. Bhushan, M. Kumar, P. Kumar, T. Stephan, A. Shankar, and P. Liu, "FAJIT: a fuzzy-based data aggregation technique for energy efficiency in wireless sensor network," Complex \& Intelligent Systems, vol. 7, no. 2, pp. 997-1007, 2021.

[18] S. P. Singh and S. C. Sharma, "A PSO based improved localization algorithm for wireless sensor network," Wireless Personal Communications, vol. 98, no. 1, pp. 487-503, 2018.

[19] B. Wang, X. Gu, L. Ma, and S. Yan, "Temperature error correction based on BP neural network in meteorological wireless sensor network," International Journal of Sensor Networks, vol. 23, no. 4, pp. 265-278, 2017.

[20] T. Y. Ling and Y. C. Chiang, "Well-being, health and urban coherence-advancing vertical greening approach toward resilience: a design practice consideration," Journal of Cleaner Production, vol. 182, pp. 187-197, 2018.

[21] H. Li, Y. Liu, C. Wu, and L. Jia, "Design method of green ecological building considering light environmental pollution," Ekoloji, vol. 28, no. 108, pp. 1301-1306, 2019.

[22] H. F. Si and Z. Yang, "Research of the application of virtual reality in green construction," Advanced Materials Research, vol. 462, pp. 592-597, 2012.

[23] S. Shao, A. Khreishah, and I. Khalil, "Joint link scheduling and brightness control for greening VLC-based indoor access networks," Journal of Optical Communications and Networking, vol. 8, no. 3, pp. 148-161, 2016.

[24] O. Franek and Č. Jarský, "On implementation of plants in the indoor environment in intelligent buildings," Environmental Research, Engineering and Management, vol. 77, no. 3, pp. 66-73, 2021.

[25] S. H. Alsamhi, O. Ma, M. S. Ansari, and Q. Meng, "Greening Internet of Things for greener and smarter cities: a survey and future prospects," Telecommunication Systems, vol. 72, no. 4, pp. 609-632, 2019.

[26] X. Jing, S. Li, J. Cheng, and J. Guo, "Multidimensional situational information fusion method for energy saving on campus," Journal of Intelligent \& Fuzzy Systems, vol. 38, no. 4, pp. 4793-4807, 2020.

[27] G. S. Odiyur Vathanam, K. Kalyanasundaram, R. M. Elavarasan et al., "A review on effective use of daylight harvesting using intelligent lighting control systems for sustainable office buildings in India," Sustainability, vol. 13, no. 9, p. 4973, 2021.

[28] Y. Bai, W. Zhang, X. Yang, S. Wei, and Y. Yu, “The framework of technical evaluation indicators for constructing low-carbon communities in China," Buildings, vol. 11, no. 10, p. 479, 2021.

[29] G. Han, Y. Wen, J. Leng, and L. Sun, "Improving comfort and health: green retrofit designs for sunken courtyards during the summer period in a subtropical climate," Buildings, vol. 11, no. 9 , p. $413,2021$. 